\title{
High-Resolution Impulse Radio Ultra Wideband Ranging
}

\author{
Hai Zhan ${ }^{1,2}$, Jaouhar Ayadi ${ }^{1}$, John Farserotu ${ }^{1}$ and Jean-Yves Le Boudec ${ }^{2}$ \\ \{hai.zhan, jaouhar.ayadi, john.farserotu\}@csem.ch, \{jean-yves.leboudec\}@epfl.ch \\ ${ }^{1}$ Centre Suissse d'Electronique et de Microtechnique SA (CSEM), Wireless Communication Section, \\ Neuchatel , Jaquet-Droz 1 - CH-2007 Neuchatel , Switzerland
${ }^{2}$ Ecole Polytechnique Fédérale de Lausanne (EPFL), School of Computer and Communication Sciences, LCA2, Ecublens, CH-1015 Lausanne, Switzerland

\begin{abstract}
We propose a high resolution ranging algorithm for unsynchronized impulse radio Ultra-wideband (UWB) communication systems in gaussian noise. We pose the ranging problem as a Maximum Likelihood (ML) estimation problem for the channel delays and attenuations and phase offset at receiver. Then we translate the obtained delay estimates into an estimate of the distance. The ML problem is very non linear and hard to solve. Some previous works focused on finding alternative estimation procedures, for example by denoising. In contrast, we tackle the ML estimation problem directly. First, we use the same transformation as the first step of Iterative Quadratic Maximum Likelihood (IQML) and transform the ML problem into another optimization problem that gets rid of the amplitude coefficients. Second, we solve the remaining optimization problem with a gradient descent approach ("pseudo-quadratic maximum likelihood"). We show that our algorithm performs significantly better than previously published heuristics. We tested the approach on a real non-line of sight system and obtained good accuracy.
\end{abstract}

Index Terms - maximum likelihood estimator, pseudoquadratic maximum likelihood, impulse radio ultra-wideband

\section{INTRODUCTION}

Ultra-wideband (UWB) radio, a new carrierless communication scheme using impulses, is a candidate technology for future communication and ranging applications. Recent progress on both in the technical and regulatory side of this technology has made this possible. The fine time resolution and material penetration capability of UWB signals has created a vision of novel ranging and positioning applications to augment existing narrowband systems operating in dense multipath environments.

We propose a high resolution Impulse Radio UWB ranging algorithm in this paper. We assume that ranging is performed by a combination of message exchanges, as for example discussed in [7]. As a result of such procedures, the receiver expects a signal from the source within a short window of time. The problem then becomes for the receiver to accurately estimate, using its own clock reference, the time of arrival of the expected signal. The signal is a pulse distorted by a multipath channel. We are searching for a high accuracy solution that works even in light non line of sight, for example with obstruction due to small objects. In Section II we argue that, within this setting, the frequency offset between source and destination does not need to be estimated (but it must be estimated at a higher level using differential procedures [7]).

We proposed a high resolution Impulse Radio UWB ranging algorithm in this paper. First, we pose the ranging problem as a Maximum Likelihood (ML) estimation problem for the channel delays and attenuations and phase offset at receiver. Ranging is obtained by translating the obtained delay estimates into an estimate of the distance. The ML problem is very non linear and hard to solve. Some previous works focused on finding alternative estimation procedures, for example by denoising [6].

We use an alternative approach where we aim to directly solve the ML estimation as an optimization problem. This is a difficult problem in low SNR cases, or when the line of sight channel amplitude is weak (non severe non line of sight). A direct solution using classical heuristics does not perform well in such cases; indeed, the opposite of the log-likelihood has many local minima, and extensive search procedures with many iterations are required.

Our first step is to observe that if the channel delays and phase offset would be known, we would have a linear regression problem, whose solution can be expressed exactly in matrix form. This allows transforming the maximum likelihood problem into an equivalent one but of fewer dimensions (see Eq. (19)). The resulting problem fits in the class of problems solved by iterative quadratic maximum likelihood (IQML) [1]. However, we found that this iterative heuristic does not work well. Instead, it is possible to use the gradient based approach, of [3]. We find that it reaches the optimum in very few steps. Furthermore, the initialization is done implicitly (we do not need an explicit initialization using another estimation procedure). The obtained performance of the proposed ranging algorithm is quasi-optimal (since it numerically solves the exact ML problem) and its processing complexity is very low: only two iterations are required to obtain a very accurate position estimate. 
We also assume that noise is Gaussian, leaving the case of impulsive interference for further study.

Section II introduces our system model. In Section III we transform our maximum likelihood estimation problem into a pseudo-quadratic one. In Section IV we define our proposed pseudo-quadratic maximum likelihood algorithm. In Section $\mathrm{V}$ we proposed a phase offset estimation algorithm. Section VI shows simulation results and Part VII illustrates the performance on a real testbed with non line of sight.

\section{SYSTEM MODEL}

\section{A. Time Domain}

Consider a multipath channel with finite delay spread and an impulse response given by

$$
c(t)=\sum_{l=1}^{L} a_{l} \delta\left(t-\tau_{l}\right)
$$

where $a_{l}$ and $\tau_{l}$ denote the amplitude and the time of arrival (TOA) of the $L$ channel paths, respectively. We take as reference the clock of the receiver and the main goal is to estimate $\tau_{1}$ (but for this we will need to estimate all parameters).

Assume that a sequence of Ultra wideband pulses with waveform, $w(t)$ of duration $T_{p}$, is received through this channel. The received signal can be expressed as

$$
y(t)=\sum_{l=1}^{L} a_{l} w\left(r \cdot\left(t-\tau_{l}\right)\right)+n(t)
$$

where $r$ accounts for the clock difference between sender and receiver (assumed constant for the duration of the reception) and $n(t)$ is an additive independent white Gaussian noise with zero mean and variance equal to $\sigma_{n}^{2}$.

We are interested in the joint estimation of the time of arrival of the different paths based on the observation in the interval $[0, T]$. Define $\vec{\tau}=\left[\tau_{1}, \tau_{2}, \cdots, \tau_{L}\right]^{T} \quad$ and $\vec{a}=\left[a_{1}, a_{2}, \cdots, a_{L}\right]^{T}$ as the vectors concatenating the channel time delays and amplitudes, respectively and where the superscript ${ }^{T}$ refers to the transpose operator. We also assume $0<\tau_{1}<\tau_{2}<\cdots<\tau_{L}$ and $\max _{l}\left\{\tau_{l}\right\} \leq T-T_{p}$.

The receiver knows the theoretical waveform $w(t)$ but not the channel parameters. The receiver reproduce the transmit waveform $w_{1}(t)=w\left(t-t_{h}\right)$. We define $t_{h}$ is the phase offset. Hence Eq.(2) becomes:

$$
y(t)=\sum_{l=1}^{L} a_{l} w_{1}\left(r \cdot\left(t-\tau_{l}+t_{h}\right)\right)+n(t)
$$

We assume that, as a result of a measurement procedure, the receiver expects receiving a (distorted) sequence over a window of the order of a microsecond. The received signal is collected in $M$ samples at time instants $t_{i}=i T_{s}(i=1,2, \cdots, M)$ the where $T_{s}$ denotes the sampling interval. The number of samples $M$ is of the order of a thousand; further, we assume the clock accuracies are order of $20 \mathrm{ppm}$. As a result, during one thousand samples, the phase shift varies in by at most $0.04 T_{s}$, which we consider negligible. We leave it for further study to extend our model to very long ranging signals (thus large values of $M$ ) or very low clock accuracy that would require estimating $r$. Therefore, we set $r$ to 1 in the Eq.(2) and Eq.(3).

The $M$ samples are concatenated in the vector $\vec{y}=\left[y_{1}, y_{2}, \cdots, y_{M}\right]^{T}, y_{i}=y\left(i T_{s}\right)$ for $i=1,2, \cdots, M$ ( $M$ satisfies $T=M T_{s}$ ) through the transmission of $K$ samples forming the UWB pulse $\left(T_{p}=K T_{s}\right.$ and $\left.K<M\right)$. The discrete version of the time delay $\tau_{l}$ that satisfies $\tau_{l} \cong D_{l} T_{s}$ and define $\vec{D}=\left[D_{1}, D_{2}, \cdots, D_{L}\right]^{T}$.The discrete version of the phase offset $t_{h}$ that satisfies $t_{h} \cong J T_{s}$. Hence, the samples of the received signal can be expressed as:

$$
y_{i}=\sum_{l=1}^{L} a_{l} w_{1}\left(i T_{s}-D_{l} T_{s}+J T_{s}\right)+n\left(i T_{s}+J T_{s}\right)
$$

\section{B. Frequency Domain}

Assuming that $N$ samples of the Discrete Fourier transform are available at the set of frequencies $\left\{f_{1}, f_{2}, \cdots, f_{N}\right\}$ such that $f_{k}=\frac{k-1}{N}$ and $k=1, \cdots, N$., we obtain

$$
Y\left(f_{k}\right)=\sum_{l=1}^{L} a_{l} W_{1}\left(f_{k}\right) e^{j f_{k} J} e^{-j f_{k} D_{l}}+N\left(f_{k}\right)
$$

where $W_{1}\left(f_{k}\right)$ and $N\left(f_{k}\right)$ are the Fourier transform of $\vec{w}_{1}=\left[w_{1}\left(T_{s}\right), w_{1}\left(2 T_{s}\right), \cdots, w_{1}\left(K T_{s}\right)\right]$ and $\vec{n}=\left[n_{1}, n_{2}, \cdots, n_{M}\right]^{T}, n_{i}=n\left(i T_{s}+J T_{s}\right)$ respectively.

We can write Eq.(5) in matrix form:

$$
\vec{Y}=U(J) S V(\vec{D}) \vec{a}+\vec{N}
$$

where $U(J)$ and $S$ are an $N \times N$ diagonal matrixes and $V(\vec{D})$ is an $N \times L$ matrix, defined as 


$$
\begin{aligned}
& U(J)=\left[\begin{array}{cccc}
1 & & & \\
& e^{j \frac{2 \pi t J}{N}} & & \\
& & \ddots & \\
& & & e^{j \frac{2 \pi(N-1) J}{N}}
\end{array}\right] \\
& S=\left[\begin{array}{lll}
W_{1}\left(f_{1}\right) & & \\
& \ddots & \\
& & W_{1}\left(f_{N}\right)
\end{array}\right] \\
& V(\vec{D})=\left[\vec{v}\left(D_{1}\right), \vec{v}\left(D_{2}\right), \cdots, \vec{v}\left(D_{L}\right)\right] \\
& \vec{v}\left(D_{k}\right)=\left[1, e^{-j \frac{2 \pi D_{k}}{N}}, e^{-j \frac{2 \pi 2 D_{k}}{N}}, \cdots, e^{-j \frac{2 \pi(N-1) D_{k}}{N}}\right]^{T}
\end{aligned}
$$

In the Eq.(8), the diagonal elements of the matrix $S$ corresponds to the Fourier transform of the UWB pulse, $\vec{W}_{1}(f)$, taken at the set of frequencies $\left\{f_{1}, f_{2}, \cdots, f_{N}\right\}$. In the Eq.(6), $\vec{N}$ is due to noise and to the discrete effect of the Fourier transform. As the noise is assumed to be additive, independent, white and Gaussian, the elements of $\vec{N}$ will be additive Gaussian variables.

\section{REDUCTION OF THE ML ESTIMATION PROBLEM}

Our problem is how to find the ML estimator of $\vec{\tau}$ and $\vec{a}$. When the observation noise is Gaussian, the Maximum Likelihood (ML) criterion becomes equivalent to a Least Squares (LS) one.

First, we assume $J$ at receiver is known. (In section $\mathrm{V}$, we will explain how to estimate $J$.) Using the deterministic ML approach, the cost function to be minimized is given by the following LS criterion

$$
F_{M L}(J, \vec{D}, \vec{a})=\|\vec{Y}-U(J) S V(\vec{D}) \vec{a}\|_{F}^{2}
$$

We get the ML estimator of $\vec{\tau}$ as follows:

$$
\hat{\vec{D}}=\arg \min _{\vec{D}}\left\{\vec{Y}^{H} P_{U(J) S V(\vec{D})}^{\perp} \vec{Y}\right\}
$$

where $P_{U(J) S V(\vec{D})}^{\perp}$ is the orthogonal projection on the subspace spanned by $U(J) S V(\vec{D})$ and is given by $I-P_{U(J) S V(\vec{D})}$.

If the discrete Fourier transform frequencies, $f_{k}=\frac{k-1}{N}$, are chosen, then $V(\vec{D})$ is a Vanderonde matrix. Hence, there exist a Toeplitz matrix, $B$, of dimension $N \times(N-L)$ such that

$$
B^{H} V(\vec{D})=0
$$

The matrix $B$ is given by

$$
B=\left[\begin{array}{cccc}
b_{0} & 0 & & \\
b_{1} & b_{0} & \ddots & \\
\vdots & b_{1} & \ddots & 0 \\
b_{L} & \vdots & \ddots & b_{0} \\
0 & b_{L} & \ddots & b_{1} \\
& \ddots & \ddots & \vdots \\
& & 0 & b_{L}
\end{array}\right]
$$

and its elements are taken from the coefficients of polynomial

$$
b(z)=b_{0} z^{L}+b_{1} z^{L-1}+\cdots+b_{L}
$$

with roots equal to $\left\{e^{-j \frac{2 \pi D_{1}}{N}}, e^{-j \frac{2 \pi D_{2}}{N}}, \cdots, e^{-j \frac{2 \pi D_{L}}{N}}\right\}$. Since the previous roots are on the unit circle, the coefficient vector $\vec{b}=\left[b_{0}, b_{1}, \cdots, b_{L}\right]^{T}$ can be assumed to be conjugating symmetric. From (14), it is clear that if $S\left(f_{k}\right) \neq 0$ for $k=1, \cdots, N$, we have

$$
B^{H} S^{-1} U(J)^{-1} U(J) S V(\vec{D})=0
$$

And hence the column space of $U(J) S V(\vec{D})$ is orthogonal to the column space of $U(J)^{-H} S^{-H} B$. Thus

$$
P_{U(J) S V(\vec{D})}^{\perp}=P_{U(J)^{-H} S^{-H} B}
$$

and hence the ML criterion given in Eq.(11) can be reformulated in terms of $\vec{b}$ rather than $\vec{D}$ as

$\hat{\vec{b}}=\arg \min _{\vec{b}}\left\{\vec{Y}^{H} S^{-H} U(J)^{-H} B R^{-1} B^{H} U(J)^{-1} S^{-1} \vec{Y}\right\}$

where $R=B^{H} S^{-1} S^{-H} B$.Since $B^{H}$ is linear in $b^{*}$ (where the superscript ${ }^{*}$ refers to the complex conjugate operation), a matrix $Z$ filled out with the elements of the vector $\vec{Z}=U(J)^{-1} S^{-1} \vec{Y}$, can be found such that $Z \vec{b}^{*}=B^{H} \vec{Z}$, Eq. (18) becomes:

$$
\hat{\vec{b}}=\arg \min _{\vec{b}}\left\{\vec{b}^{H} Z^{T} R^{-T} Z^{*} \vec{b}\right\}
$$

To find the ML estimator of $\vec{D}$ is equivalent to find $\vec{b}$. In iterative quadratic maximum likelihood (IQML) algorithm [1], the search $\vec{b}$ is replaced by an iterative procedure. For a given IQML iteration, the vector $\vec{b}$ in $R^{-T}$ is held fixed, and 
the Eq.(19) is then quadratic in the remaining involving $\vec{b}$ and can be minimized in closed-form. The resulting $\vec{b}$ is then used to fix $R^{-T}$, and the process is repeated until $\vec{b}$ converges. IQML algorithm leads to good estimates for high values of the Signal to Noise Ratio (SNR). At low values of the SNR, the IQML approach diverge and the global ML cost function minimum is not found. In the next part, we used pseudo-quadratic maximum likelihood (PQML) algorithm to find the global ML cost function minimum in the low SNR environments.

\section{PSEUDO-QUADRATIC MAXIMUM LIKELIHOOD TOA ESTIMATION}

In [2][3], the PQML approach was shown to outperform proposed ML based multichannel estimation techniques and to lead to the ML cost function minimum. We propose here to apply this technique to the TOA estimation case. The main idea is to arrange the gradient of the ML cost function as $\Gamma(\vec{b}) \vec{b}$, where $\Gamma(\vec{b})$ is (ideally) positive semi-definite. The ML solution verifies $\Gamma(\vec{b}) \vec{b}=0$ and is solved under the following PQML approach: in a first step $\Gamma(\vec{b})$ is considered constant, and since $\Gamma(\vec{b})$ is positive semi-definite, the minimization problem becomes quadratic. Hence $\vec{b}$ can be estimated from such quadratic criterion and its estimate can be refined iteratively. As mentioned in [3] for multichannel estimation case, the key issue is to find the appropriate $\Gamma(\vec{b})$ and especially with the positive semi-definite constraint. For our TOA estimation problem, the gradient of the ML cost function given by Eq.(18), can be got from the following process:

$$
\begin{aligned}
& \frac{\partial}{\partial b_{i}}\left\{\vec{Y}^{H} S^{-H} U(J)^{-H} B R^{-1} B^{H} U(J)^{-1} S^{-1} \vec{Y}\right\}= \\
& \frac{\partial}{\partial b_{i}} \vec{b}^{H}\left\{Z^{T} R^{-T} Z^{*}-\Psi^{H} S^{-1} S^{-H} \Psi\right\} \vec{b}
\end{aligned}
$$$$
\text { So } \Gamma(\vec{b})=Z^{T} R^{-T} Z^{*}-\Psi^{H} S^{-1} S^{-H} \Psi
$$

where the matrix $\Psi$ is defined as $T=R^{-1} B^{H} \vec{Z}$ and $B T=\Psi \vec{b}$.

Asymptotically, the effect of the second term is to remove the noise contribution present in the first one, then $\Gamma(\vec{b})=X^{T} R^{-T} X^{*}$. Any initialization of $\Gamma(\vec{b})$ gives a consistent estimate for the vector $\vec{b}$ and the second iteration converges to the global minimum.
For finite $N$, the matrix $\Gamma(\vec{b})$ is indefinite and applying directly the PQML strategy will not work except for high SNR. We introduce an arbitrary $\lambda$ in the minimization criterion, which becomes:

$$
\hat{\vec{b}}=\arg \min _{\vec{b}} \vec{b}^{H}\left\{Z^{T} R^{-T} Z^{*}-\lambda \Psi^{H} S^{-1} S^{-H} \Psi\right\} \vec{b}
$$

with semi-definite positive constraint on the central matrix. Hence, $\vec{b}$ can be found as the minimal generalized eigenvector of $Z^{T} R^{-T} Z^{*}$ and $\Psi^{H} S^{-1} S^{-H} \Psi . \lambda$ is the corresponding minimum generalized eigenvalue. Asymptotically, there is a global convergence for $\vec{b}$, which converges to the ML minimum and for $\lambda$, which converges to 1. Note also that the stationary points of PQML are the same as those of ML criterion; this is why PQML has the same performance as the ML criterion and hence gives the global ML minimum.

\section{PHASE OFFSET ESTIMATION AND HIGH- RESOLUTION PQML ALGORITHM}

From $\vec{Z}=U(J)^{-1} S^{-1} \vec{Y}$ and Eq.(22), we know the PQML algorithm can not estimate $\vec{D}$ except we know $J$.

Clearly from Eq.(5), in order to completely characterize the channel, we need to estimate $a_{l}$ 's and $D_{l}$ as well as $J$, which, in general, requires a non-linear estimation procedure. However, one possible way to obtain a closed form solution is to approximate $e^{j f_{k} J}$ with polynomials of degree $D \leq G-1$,that is,

$$
e^{i f_{k} J}=\sum_{g=0}^{G-1} p_{g}(k-1)^{g}
$$

So we can get:

$$
\sum_{g=0}^{G-1} p_{g}(k-1)^{g} \cdot e^{-j f_{k} D_{l}}=e^{-j f_{k}\left(D_{l}-J\right)}
$$

We know we can estimate $D_{l}-J$ from PQML algorithm. So, there are only $p_{g}$ and $D_{l}$ are unknown parameters in Eq.(24). If the number of unknown parameters $(G+1)$ is not larger than $N$, we can get $p_{g}$ and $D_{l}$.

We briefly summarize our high-resolution PQML(HPQML) algorithm as following:

1. Given a set of Discrete Fourier transform $\left\{Y\left(f_{k}\right)\right\}$ and $\left\{W_{1}\left(f_{k}\right)\right\}$. 
2. Set $q=0, J_{s}=0$, the number of iterations $Q$, matrix $R$ equal to identity matrix and The degree of polynomial $G-1$ in Eq.(23).

3. Compute $\vec{b}_{q}$ which is equal to the minimal eigenvector of $Z^{T} Z^{*}$ (Eq.(19)). We can get $\vec{d}_{q}$ (Assume $\vec{d}=\vec{D}-J$ )with $\vec{b}_{q}$.

4. Compute $p_{g}$ with $\vec{d}_{q}$ (Eq.(24)) and compute $J_{q}$ (Eq.(23)) and so $\vec{\tau}_{q}=\left(\vec{d}_{q}+J_{s}\right) \cdot T_{s}$

5. $q=q+1$

6. Compute matrix $Z^{T} R^{-T} Z^{*}$ and $\Psi^{H} S^{-1} S^{-H} \Psi$ with $\vec{b}_{q-1} \cdot \vec{b}_{q}$ is equal to the minimal eigenvector of these two matrixes (Eq.(22)). We can get $\vec{d}_{q}$ with $\vec{b}_{q}$

7. Compute $p_{g}$ with $\vec{d}_{q}$ (Eq.(24)) and compute $J_{q}$ (Eq.(23)) and so $\vec{\tau}_{q}=\left(\vec{d}_{q}+J_{q}\right) \cdot T_{s}$

8. If $q<=Q$,go back to step 5. Otherwise, stop iteration.

\section{SIMULATIONS}

We consider a received UWB signal composed of Impulse Radio (IR) pulses. Each pulse has duration $1 \mathrm{~ns}$ in a total burst length of 100ns, which means 1 percent duty cycle. On the IR UWB pulse, we apply an FFT of length $\mathrm{N}=128$. The sampling frequency is $2 \mathrm{GHz}$. The used channel is a five-ray propagation channel with path amplitudes equal to [0.153 $\left.\begin{array}{llll}0.7468 & -0.4451 & 0.9318 & 0.4660\end{array}\right] \quad$ and

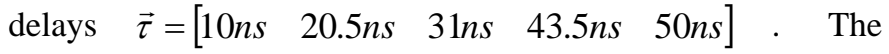
sampling offset at receiver is $3.6 \mathrm{~ns}$. The degree of polynomial $G-1$ in Eq.(21) is set to 2 . All the presented results are average over 100 Monte-Carlo trials and for a given trial the Normalized Minimum Square Error(NMSE) for a vector of parameters is defined by $\|\vec{u}-\hat{\vec{u}}\|_{F}^{2} /\|\vec{u}\|_{F}^{2}$, and $\hat{\vec{u}}$ refers to the vector of parameters estimate. The initialization of the

UWB TOA PQML algorithm is done by putting the matrix $R$ equal to identity. Note that this initialization corresponds to the solution of the following minimization LS problem $\min _{\vec{b}}\left\|S^{-H} B \vec{Y}\right\|_{F}^{2}$ and that the ML criterion given in Eq.(17) corresponds to its optimally weighted LS version.

We consider now a Gaussian pulse generated with a center frequency $f_{c}=5.12 \mathrm{GHz}$, according to the following shape: $w(t)=4 \pi \sqrt{2.7} f_{c} e^{-2\left(\pi f_{c} t\right)^{2}}$ ( the duration and duty cycles are as previously described). Figure 1 illustrates the obtained
NMSE versus the number of iterations at different values of SNR. NMSE of Channel Amplitude Estimation versus the number of iterations at different values of SNR is showed in Figure 2. We can note that there is a significant performance improvement when using the UWB TOA HPQML estimation algorithm. Figure 3 compare the UWB TOA HPQML estimation algorithm with subspace algorithm which is proposed in [6]. We can find the algorithm works better than the subspace algorithm.

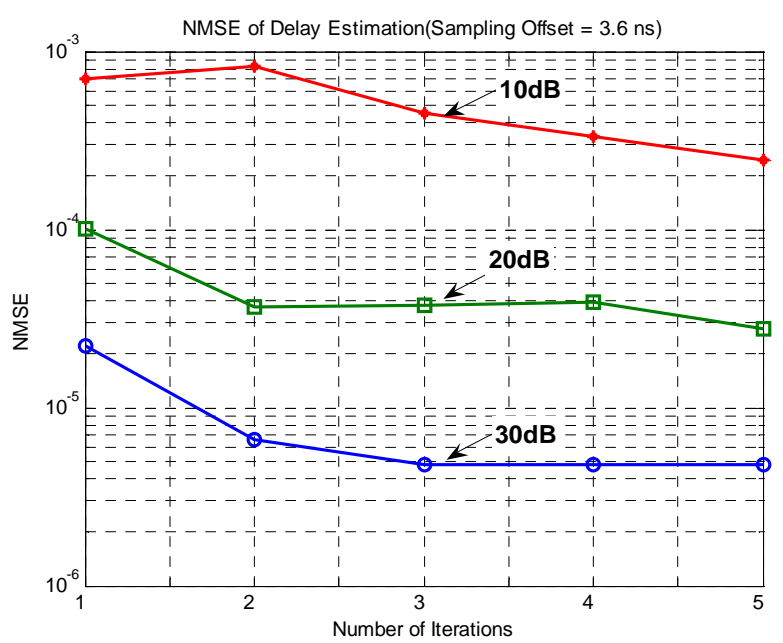

Fig. 1. Performance of PQML UWB TOA

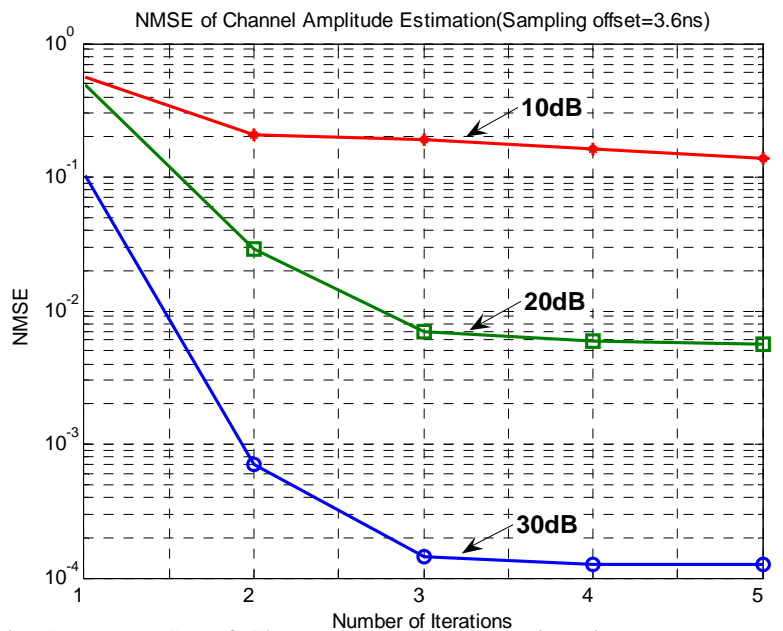

Fig. 2. NMSE of Channel Amplitude Estimation

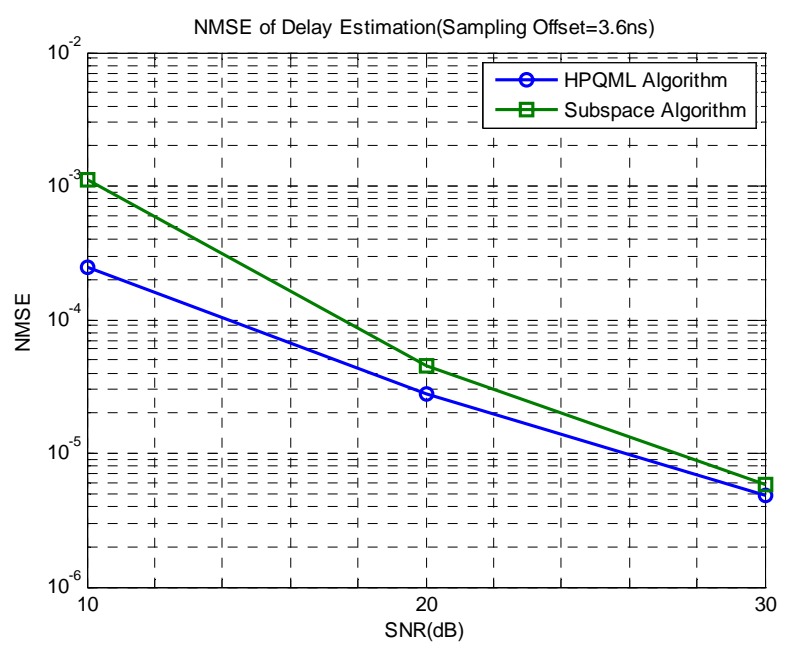


Fig. 3. NMSE comparison between HPQML and Subspace

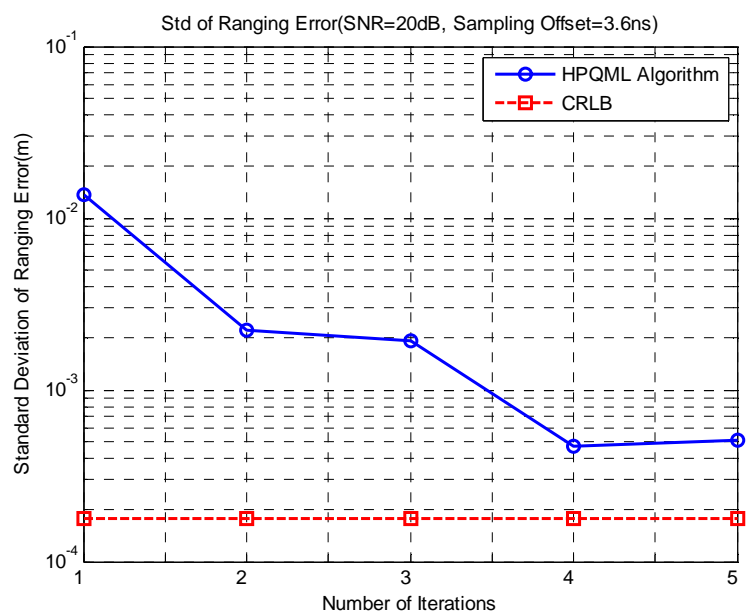

Fig. 4. Standard Deviation of Ranging Error of HPQML TOA

Coming now to the ranging, we consider only the first path, $\tau_{1}$ form the vector $\vec{\tau}$ as the estimated parameter. Figure 4 illustrates the obtained performance in terms of standard deviation of the ranging error and compares it to its CramerRao Lower Bound (CRLB). We can conclude that using the HPQML algorithm for the estimation of the UWB TOA and then translating this to a ranging estimation leads to good results for the UWB ranging. From the above simulation results, the PQML algorithm is better than Subspace algorithm. The reason is the PQML algorithm is based on Maximum Likelihood Estimation estimator which is optimal estimator under Gaussian noise environment.

\section{INTEGRATION WITH MICS UWB TESTBED}

The Swiss project MICS developed an UWB testbed at EPFL recently. We used measurements from this testbed to verify the algorithm. To get Non-line of sight signal, we put one book between the transmitter and receiver. The distance between the transmitter and the receiver is about $34.8 \mathrm{~cm}$. The sampling frequency is $1 \mathrm{GHz}$. We assume the number of paths is 41 .

One FPGA box generates one signal to trigger one pulse. The duration of this pulse is about 1 ns. After passing one filter and antenna, the pulse is transmitted to the receiver. The transmitted and received signal are as follows:
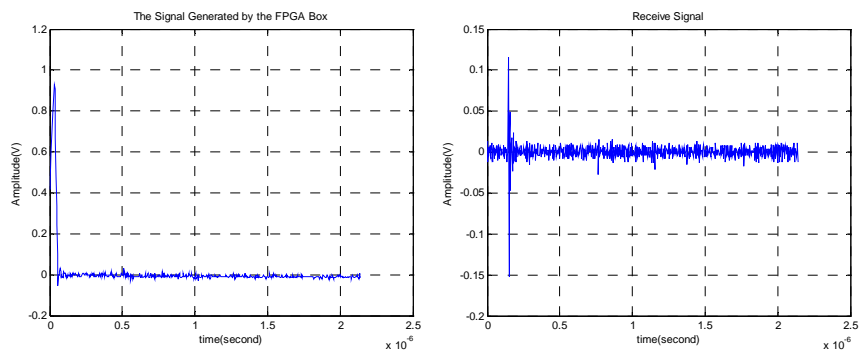

Fig. 5. (a)The Transmitted signal (b) Received Signal

The transmitter sends the pulse train. We feed the first transmit signal into the algorithm. The estimate distance is $32.6 \mathrm{~cm}$.

\section{CONCLUSIONS}

The developed algorithm relies on the exploitation of the UWB nature of the transmitted signal in combination with an efficient denoising procedure of the maximum likelihood cost function. This joint combination leads to spectacular enhancement in the estimation of the Time of Arrival (TOA) of the received UWB signal, which immediately translates to enhancement of the ranging error. Motivated by the good results that we obtained for the ranging algorithm, we investigated related reduced-complexity version, which brought us to attractive and flexible FPGA implementation. Such implementation is important in the sense it can be envisaged for tiny sensing devices such as sensors and tags, which makes our reduced-complexity version of the ranging algorithm a good candidate for ranging and proximity ranging in wireless sensors based applications. In the future, we will also consider the cases of (1) non Gaussian, impulsive interference noise and (2) long signals that improve the estimation but require estimating the clock frequency differences.

\section{REFERENCES}

[1] A. L. Swindlehurst and J. H. Gunther. Methods for Blind Equalization and Resolution of Overlapping Echoes of Unknown Shape. In IEEE Transactions on Signal Processing, Vol. 47, No. 5, 1999.

[2] J. Ayadi, H. Zhan, J. Farserotu. Maximum Likelihood Time of Arrival Esitmation for Ultrawideband Signals. International Conference on Information Sciences, Signal Processing and its Applications, Sharjah, United Arab Emirates, 12-15 February,2007

[3] J. Ayadi, E. de Carvalho,D.T.M. Slock. Blind and Semiblind Maximum Likelihood Methods for FIR Multichannel Identification. In Proc. ICASSP 98 Conf., USA, May 1998.

[4] Joon-Yong Lee and Roben A.Scholtz, Ranging in a Dense Multipath Environment Using an UWB Radio Link, IEEE Journal on selected areas in communications. VOL20.NO.9, December 2002.

[5] C. Falsi, D. Dardari, L. Mucchi and M. Z. Win. Time of Arrival Esitmation for UWB Localizers in Realistic Environments.In Journal on Applied Signal Processing, Vol. 2006, pp1-13, 2006

[6] Irena Marvais, Sampling Methods For Parametric Nonbandlimited Signals: Entensions And Applications, Ph.D thesis, EPFL,2004

[7] B.Zhen,H.B. Li, R. Kohno, Clock Offset Compensation in UltraWideband Ranging, IEICE Trans. Fundamentals,Vol.E89-

A.NO.11 November 2006 
